\title{
Retention and Stability- A Review
}

\author{
N Raj Vikram \\ Research Scholar, Dr. Mgr Educational Research Institute University, India
}

Submission: January 03, 2019; Published: January 22, 2019

*Corresponding author: N Raj Vikram, Research Scholar, Thai Moogambigai Dental College And Hospital, Dr. Mgr Educational Research Institute University, Chennai, India

\begin{abstract}
The stability of treatment outcome in orthodontics remains a fundamental issue of concern and debate. Usually a retention phase is required after active Orthodontic tooth movement to maintain the ideal aesthetic and functional relation and prevent the teeth to return to their former position. In the importance of retention in orthodontic treatment, this article makes an attempt to refresh our knowledge on retainers by exploring the literature.
\end{abstract}

Keyword: Retention; Retainers; Relapse; Stability; Functional relation

\section{Introduction}

In Orthodontics the stability of the achieved result remains a fundamental issue of concern and debate. Tirk has said "The result of Orthodontic therapy - good, bad or indifferent is only evident many years out of retention". Maintaining teeth in their corrected positions after Orthodontic treatment has been continuous to be the challenge [1]. A phase of retention is normally required after active Orthodontic tooth movement to hold teeth in ideal aesthetic and functional relation and combat the inherent tendency of the teeth to return to their former position [2]. Stability can only be achieved if the forces derived from the periodontal and gingival tissues, the orofacial soft tissues, the occlusal forces and post treatment facial growth and development are in equilibrium [3].

\section{Retainers}

The retainers in Orthodontics can be chiefly classified into Removable and Fixed retainers and visible a and invisible retainers.

\section{Removable Retainers}

The removable retainers serve as retention for intra-arch stability and are useful as retainers in patients with growth problems. The available removable retainers are discussed briefly

\section{Hawley's retainer [4]}

The most commonly used retainer designed in 1920's as an active removable appliance. It incorporates clasps on molar teeth and a characteristic outer labial bow with adjustment loops, spanning from canine to canine. The outer bow provides excellent control of the incisors even if it is not adjusted to retract them. When first premolars are extracted, one function of a retainer is to keep the extraction space closed, which the standard design cannot do. A common modification of the Hawley retainer for use in extraction cases is a bow soldered to the buccal section of Adam's clasp on the first molars, so that the action of the bow helps hold the extraction site closed.

\section{Removable wrap around retainer [4]}

The wrap around or clip-on retainer, which consists of a plastic bar along labial and lingual surfaces of teeth. A full arch wrap around retainer should allow each tooth to move individually, stimulating reorganization of the periodontal ligament. In addition, a wraparound retainer, though quite aesthetic, is often less comfortable than a Hawley retainer and may not be effective in maintaining overbite correction. A full arch wrap around retainer is indicated primarily when periodontal breakdown requires splinting of teeth together.

\section{Removable cuspid to cuspid retainer [5]}

These types of retainers can be made relatively quickly and by untrained personnel. The construction of the appliance is with the use of two 0.025 " wires, which are bent and placed in the embrasure between the lower cuspid and lateral incisors and a separating medium is placed on the cast. The salt and pepper method are used to apply a quick- cure acrylic covering the labial surfaces from cuspid to cuspid and the lingual surfaces from first bicuspid to first bicuspid or second bicuspid if first bicuspids have been extracted. When the acrylic has set, retainer is removed from the cast, trimmed, pumiced and polished. The acrylic should be cut down on the labial to avoid being struck by the maxillary central incisors but left at the incisal edge on 
the lingual. The whole procedure requires about fifteen minutes of working time and produces a smooth, inconspicuous, easily fitted retainer that will do a positive job of controlling corrected lower rotations.

\section{Removable 6-6 Metal retainer [6]}

The lingual arch is formed of $0.045^{\prime \prime}$ hard SS wire. Adams clasps are formed of 0.028 or 0.035 " SS wire. Bend clasp tails over arch wire so that stress is wire to wire and not on solder. In soldering, use Hydro flame or electro soldering. Add buccal wires, tubes, lingual finger springs, ball end clasps, and distal extensions to second molars.

\section{Positioners as retainers [4]}

A tooth positioner can also be used as a removable retainer either fabricated for this purpose alone, or more commonly, continued as a retainer after serving initially as a finishing device. Positioners are excellent finishing devices and under special circumstances can be used to an advantage as retainers. For routine use, however, a positioner does not make a good retainer. The major problems with positioners as retainer are as follows.

i. The pattern of wear of a positioner does not match the pattern usually desired for retainers. Because of its bulk, patients often have difficulty in wearing full time or nearly so.

ii. Positioners do not retain incisor irregularities and rotations as well as standard retainers. Also, overbite tends to increase while a positioner is being worn.

The positioner has one advantage over a standard removable or wrap around retainer - it maintains the occlusal relationships as well as intra-arch tooth positioners. For a patient with a tendency towards class III relapse, a positioner made with the jaws rotated somewhat downward and backward may be useful [7].

\section{Thermoplastic essix retainers [4]}

Essix thermoplastic copolyester retainers are a thinner, but stronger, cuspid to cuspid version of the full arch, vacuum formed devices. Essix retainers can be placed on the same day the fixed appliances are removed. The vinyl polysiloxane impression is taken immediately after debonding. Minor incisor rotations can be corrected by altering the cast, since the teeth will be slightly mobile. Seat the retainers over the incisors with firm finger pressure. If a retainer will not seat properly, it is usually because of internal plastic ridges formed by interproximal undercuts that were not adequately reduced. These ridges can be smoothed out at chair side with a scalpel. It is critical that the retainer does not slip easily over the teeth but requires a reasonable amount of pressure to flex over the interproximal undercuts gingival to the Contact points.

\section{Fixed Retainers [4]}

They are normally used in situations where intra-arch instability is anticipated and prolonged retention is planned, especially the mandibular incisor area. There are mainly four major indications, they include the following.

\section{Maintenance of lower incisor position}

An excellent retainer to hold these teeth in alignment is a fixed lingual bar, attached only to the canines and resting against the flat surface of the lower incisors above the cingulum. This prevents the incisors from moving lingually and is reasonably effective in maintaining correction of rotations in the segment. Fixed canine to canine retainers must be made from a wire heavy enough to resist distortion over the long span between these teeth. Usually 28 or 30 mil stainless steel is used for this purpose with loop bend in the end of the wire to improve retention $[7,8]$

\section{Diastema maintenance}

A second indication for a fixed retainer is a situation where the teeth must be permanently or semi permanently bonded together to maintain the closure of space between them. This is encountered most commonly when a diastema between maxillary central incisors has been closed. Even if frenectomy has been done, there is a tendency for a small space to open up between the upper central incisors. The best retainer for this purpose is a bonded section of flexible wire. The wire should be contoured so that it lies near the cingulum to keep it out of occlusal contact. The object of the retainer is to hold the teeth together while allowing some ability to move independently during function. An alternative is a solid wire to avoid the tooth contacts to facilitate flossing, which also can incorporate stops to prevent deepening of the bite.

\section{Keeping extraction space closed in adults}

A fixed retainer is both more reliable and better tolerated than a full time removable retainer and spaces reopen unless a retainer is worn consistently [9]. Bonded flexible wire lingual retainer and the flexible spiral wire retainers were found to be excellent in the following indication:

i. In midline diastema cases

ii. Spaced anterior teeth

iii. Adult cases with potential post orthodontic tooth migration

iv. Accelerated loss of maxillary incisors, requiring the closure and retention of large anterior space.

v. Severely rotated tooth.

\section{Crozat retainer [10]}

A 4-4 Crozat appliance has cribs on the first bicuspids, recurved double lapping lingual finger springs and a labial 
bow. It combines the advantages of other types of retainers and has been well received by patients. Its advantages include the following.

i. Firm retention, because of the Crozat clasping mechanism.

ii. Labiolingual control of anterior teeth to maintain or restore arch form in the lower or upper arch

iii. Flexible, because it is all wire. It can be left out for months and still fit

iv. Maintenance of adequate oral hygiene, because it is removable.

v. Esthetic, because only a single labial wire shows.

The major disadvantages of the appliance are:

i. it must be fabricated at a quality laboratory, making it cost prohibitive and

ii. it is breakable.

\section{Molar to molar mandibular retainer [11]}

The molar to molar mandibular retainer is done with the heavy gauge wire and with the use of molar bands. The advantages of molar to molar mandibular retainer over a Hawley's or a cuspid to cuspid retainer include the following.

i. Allows the mandibular canines and molars to settle naturally.

ii. Mandibular arch can be expanded or contracted

iii. Rotations can be corrected by ligating the teeth to the lingual arch

\section{Resin fiberglass bonded retainer [12]}

The Resin fiberglass bonded retainer was developed by Michael. It is a direct technique that solves the major problem with cuspid to cuspid retainer and takes 20 minutes or less with previsit preparation. The system uses glass fiber from woven fiberglass fabric or fiber bond. The main advantage of the resin fiberglass retainer have proven rigid and impervious. Patients appreciate the tooth colored material and the comfort that is provided by smoothing the margins with rubber abrasive points or wheels.

\section{Conclusion}

Retention is the important phase in orthodontic treatment that is essential to achieve post treatment Stability and its mandatory to plan the requirements of retention at the time of diagnosis and treatment planning. Permanent retention is the only way to ensure long term post treatment stability. However, as trained orthodontist it is necessary to take a more pro-active approach in dealing with the actions associated with relapse. We should aim to remove the primary burden of preventing relapse from our patients and would be well advised to maintain as treatment goals the following well documented basic principles: [1]

i. The patient's pre-treatment lower arch form should be maintained during orthodontic treatment as much as possible.

ii. Original lower intercanine width should be maintained as much as possible because of lower intercanine width is the most predictable of all orthodontic relapse.

iii. Mandibular arch length decreases with time.

iv. The most stable position of the lower incisor is its pretreatment position. Advancing the lower incisors is unstable and should be considersd as seriously compromising lower anterior post- treatment stability.

v. Fiberotomy is an effective means of reducing rotational relapse.

vi. Lower incisor reproximation shows long-term improvements in post-treatment stability.

\section{References}

1. Burke S, Silveira AM, Goldsmith LJ, Yancey JM, Van Stewart A, et al (1998) A meta-analysis of mandibular intercanine width in treatment and postretention. Angle Orthod 68(1): 53-60.

2. Blake M, Bibby K (1998) Retention and stability: a review of the literature. Am J Orthod Dentofacial Orthop 114(3): 299-306.

3. Melrose C, Millett D (1998) Toward a perspective on orthodontic retention? Am J Orthodo Dentofacial Orthop 113(5): 507-514.

4. Proffit W, Fields H, Sarver D (2012) Contemporary orthodontics. (5 ${ }^{\text {th }}$ edn), Mosby Inc. pp. 768.

5. Knierim R (1973) Invisible lower cuspid to cuspid retainer. Angle Orthod 43(2): 218-220.

6. Hoffman CS (1973) Technic clinic. A back-action arch. J Clin Orthod $7(8): 520-521$.

7. Sheridan J, LeDoux W, McMinn R (1993) Essix retainers: fabrication and supervision for permanent retention. J clin orthod 27(1): 37-45.

8. Zachrisson B (1995) Third-generation mandibular bonded lingual 3-3 retainer. J clin orthod 29(1): 39-48.

9. Bearn D (1995) Bonded orthodontic retainers: a review. Am J Orthod Dentofacial Orthop 108(2): 207-213.

10. Owen AH (1985) 4-4 Crozat retainer. J clin orthodo 19(3): 194-197.

11. Christie TE (1985) Molar-to-molar mandibular retainer. J Clin Orthod 19(7): 500-504.

12. Diamond M (1987) Resin fiberglass bonded retainer. J clin orthodo 21(3): 182 


\section{Your next submission with Juniper Publishers will reach you the below assets}

- Quality Editorial service

- Swift Peer Review

- Reprints availability

- E-prints Service

- Manuscript Podcast for convenient understanding

- Global attainment for your research

- Manuscript accessibility in different formats ( Pdf, E-pub, Full Text, Audio)

- Unceasing customer service

Track the below URL for one-step submission https://juniperpublishers.com/online-submission.php 